\title{
GAIA Postnatally Diagnosed Congenital Microcephaly Level of Diagnostic Certainty
}

National Cancer Institute

\section{Source}

National Cancer Institute. GAIA Postnatally Diagnosed Congenital Microcephaly Level of

Diagnostic Certainty. NCI Thesaurus. Code C128694.

A classification of maternal and fetal outcomes relating to diagnosing congenital

microcephaly postnatally, developed by the Global Alignment of Immunization safety

Assessment in pregnancy, based on the extent to which the diagnosis has been

confirmed. 